TRIUMF preprint: TRI-PP-05-05

\title{
Projection Operator Formalisms and the Nuclear Shell Model
}

\author{
B.K. Jenning: \\ TRIUMF, 4004 Wesbrook Mall, Vancouver, BC, Canada V6T $2 A 3$
}

(Dated: August 13, 2018)

\begin{abstract}
The shell model solve the nuclear many-body problem in a restricted model space and takes into account the restricted nature of the space by using effective interactions and operators. In this paper two different methods for generating the effective interactions are considered. One is based on a partial solution of the Schrodinger equation (Bloch-Horowitz or the Feshbach projection formalism) and other on linear algebra (Lee-Suzuki). The two methods are derived in a parallel manner so that the difference and similarities become apparent. The connections with the renormalization group are also pointed out.
\end{abstract}

PACS numbers: 21.60.Cs,21.30.Fe

\section{INTRODUCTION}

The shell model has been in use for many years. There are two main variants. The first is due to Bloch and Horowitz [1]. This method is closely related to Feshbach projection operator formalism [2] frequently used to the justify the optical model. The renormalization group treatment of the twobody scattering due to Birse et al [3] is also closely connected to this method. The second method is due to Lee and Suzuki [4]. It is used in the no-core shell model [5] and to derive the nucleon-nucleon effective interaction $V_{\text {low } k}[\underline{6}, 7$. It is the purpose the present paper to put the differences and similarities between the two methods into sharp contrast by a parallel derivation of the two methods.

\section{THE OPERATOR $\omega$ AND ITS USES}

We start with a projection operator $P=P^{2}$ and its compliment $Q=1-P$. Following Lee-Suzuki [4] we define an operator:

$$
Q \omega P|\psi\rangle=Q|\psi\rangle
$$

While this is a common part of the Lee-Suzuki method it is rarely used in the Bloch-Horowitz method. Here we use it in both methods and as will be seen the main difference between the two methods is precisely in how $\omega$ is determined. First we note that only the off-diagonal matrix elements

*Electronic address: jennings@triumf.ca of $\omega$ are needed. In general $\omega$ is not well defined a projection operator does not have an inverse. We need independent information on $|\psi\rangle$ for example that it is a solution of the Schrodinger equation or a member of a well define set. However once $\omega$ is known, we can easily construct effective interactions and operators. For example, starting with the Schrodinger equation $H|\psi\rangle=E|\psi\rangle$ we have:

$$
\begin{aligned}
& H(P+Q \omega P)|\psi\rangle=E|\psi\rangle \\
& P H(P+Q \omega P)|\psi\rangle=E P|\psi\rangle \\
& \begin{aligned}
\left(P+P \omega^{\dagger} Q\right) H(P+Q \omega P)|\psi\rangle \\
=E\left(P+P \omega^{\dagger} Q\right)|\psi\rangle \\
\quad=E\left(P+P \omega^{\dagger} Q \omega P\right)|\psi\rangle
\end{aligned}
\end{aligned}
$$

where eq. 11 has been used to eliminate $Q|\psi\rangle$. The form, eq. 3. is the simplest and is the one universally used in the Bloch-Horowitz method. It has the advantage that $\omega$ occurs linearly. The last has the advantage of being symmetric. The states $P|\psi\rangle$ are only orthogonal with with respect to the weight function. Orthogonal functions can be obtained by defining

$$
|\phi\rangle=\sqrt{P+P \omega^{\dagger} Q \omega P}|\psi\rangle .
$$

The effective interaction is now given as (see ref. [8]):

$$
\begin{aligned}
& \frac{1}{\sqrt{P+P \omega^{\dagger} Q \omega P}}\left(P+P \omega^{\dagger} Q\right) H \\
& \times(P+Q \omega P) \frac{1}{\sqrt{P+P \omega^{\dagger} Q \omega P}}|\phi\rangle=E|\phi\rangle .(7)
\end{aligned}
$$

The equations, 3 through $[7$ are formally equivalent. Which one to use is matter of taste and 
convenience. In particular using $|\psi\rangle$ has the advantage that as we increase the model space we just add additional contributions leaving the terms already present unchanged whereas $\phi$ has to be renormalized at each stage. On the other hand the $\phi$ have the advantage that they are orthogonal (at least for some choices or $\omega$ ) and an hermitian operator is being used.

By construction, eq. 3 gives an effective interaction in the $P$ space that reproduces the $P$ space components of the wave function from the fullspace calculation. If $P$ commutes with the kinetic energy (or more generally the unperturbed Hamiltonian) then the half off shell t-matrix is also reproduced since:

$$
T\left|\psi_{P W}\right\rangle=P V|\psi\rangle=V(P+Q \omega P)|\psi\rangle
$$

where $\left|\psi_{P W}\right\rangle$ is a plane wave. Acting on the left side of the last equation with $P$ restricts the tmatrix to its low momentum components below the cut off. So we have $P T\left|\psi_{P W}\right\rangle=P V(P+$ $Q \omega P)|\psi\rangle$ and can readily identify the low energy effective potential that reproduces the half off-shell t-matrix as:

$$
V_{\mathrm{eff}}=P V(P+Q \omega P) .
$$

This will be true for either the Bloch-Horowitz or Lee-Suzuki choice of $\omega$. As we will see shortly, the Bloch-Horowitz method reproduces the fully offshell t- or k-matrix leading to the same results as Birse et al's 3] application of the renormalization group. On the other hand, in the derivation of the effective interaction, $V_{\text {low }}$, the authors only require the half-off shell t-matrix to be reproduced. That approach is equivalent [6] to the Lee-Suzuki method.

It is equally easy to generate the effective operator for a given initial operator $\Theta$ :

$$
\begin{array}{r}
\langle\psi|\Theta| \psi\rangle=\left\langle\psi\left|\left(P+P \omega^{\dagger} Q\right) \Theta(P+Q \omega P)\right| \psi\right\rangle \\
=\langle\phi| \frac{1}{\sqrt{P+P \omega^{\dagger} Q \omega P}}\left(P+P \omega^{\dagger} Q\right) \Theta \\
(P+Q \omega P) \frac{1}{\sqrt{P+P \omega^{\dagger} Q \omega P}}|\phi\rangle
\end{array}
$$

As these examples illustrate $\omega$ is the key ingredient. Once it is known, the effective quantities can be calculated easily and the formal connections become clear. The great advantage of the present method using $\omega$ is that many of the derivations become very simple.

\section{THE BLOCH-HOROWITZ METHOD}

The first method for solving for $\omega$ is act on eq. 2 with $Q$, use eq. 11 and solve for $\omega$. This gives:

$$
Q \omega P|\psi\rangle=\frac{1}{E_{\psi}-Q H Q-i \epsilon} Q H P|\psi\rangle
$$

There is one aspect of this equation, that thought of as an operator equation, is quite odd (although so common that one hardly notices it anymore). Namely the right hand side depends explicitly on the energy of the state - the energy has been given a $\psi$ subscript to emphasize this point. The equation is not a general operator relation but is only valid for a limited set of matrix elements those with the given eigen function of $H$ on the right hand side. This is, presumably, the source of the starting energy problem: What energy do we use once we take this equation out of this narrow context? In spite of all that, if $|\psi\rangle$ is an eigen function of $H$ then eq. 12 must be satisfied. Even the alternate form for $\omega$ derived in the next section must obey this equation.

Eq. 12 is all that is needed to derive the effective interaction from eq. 3. Inserting eq. 12 into eq. 3 we obtain:

$$
\begin{array}{r}
\left(P H P+P H Q \frac{1}{E_{\psi}-Q H Q-i \epsilon} Q H P\right) P|\psi\rangle \\
=E P \psi(13)
\end{array}
$$

This equation can be immediately recognized as the Bloch-Horowitz shell-model equation or the Feshbach optical model equation. A bit of a miracle has occurred here since eq. 3] is asymmetric with $\omega$ appearing only on the right hand side while eq. 13 is symmetric. Nothing is gained by going to eq. 4 or 5 The effective interaction is not hermitian due to the $i \epsilon$. Since $\omega$ is energy dependent both the effective interaction and the effective operators are energy dependent. For off-diagonal matrix elements different energies and hence $\omega$ 's are needed on the left and right hand sides of eq. 10] The energy dependence of the effective interaction is also a major contributor to making the wave functions non-orthogonal. The effective interaction and operators can be used for any state with a non-zero overlap with the model space. This is in contrast to the Lee-Suzuki method discussed in the next section.

The effective interaction, eq. 13] can be derived equally well starting with the LippmannSchwinger equation $T=V+V G_{0} T$. Defining 
$T_{P}=P T P$ and $T_{Q}=Q T P$ and eliminating $T_{Q}$ gives the equation:

$$
\begin{array}{r}
T_{P}=\left(P H P+P H Q \frac{1}{E_{\psi}-Q H Q-i \epsilon} Q H P\right) \\
+\left(P H P+P H Q \frac{1}{E_{\psi}-Q H Q-i \epsilon} Q H P\right) \\
\times G_{0} T_{P}
\end{array}
$$

Thus the Bloch-Horowitz effective interaction reproduces the low momentum components of the fully off-shell t-matrix (or equivalently the $\mathrm{k}$ matrix). For two-body scattering, it reproduces the results of Birse et al $\underline{3}]$.

\section{THE LEE-SUZUKI METHOD}

The second method [4, 9] of obtaining $\omega$ is a bit more subtle. We divide the Hilbert space into two parts $|k\rangle$ and $\left|k^{\prime}\right\rangle$. For the sake of definiteness take the states to be eigenfunctions of the full Hamiltonian. Consider an orthonormal set of states, $\left|\alpha_{P}\right\rangle$, that span the $P$ space. If the states $P|k\rangle$ form a complete basis (not necessarily orthonormal) in the $P$ space then we can write any one of the $\left|\alpha_{P}\right\rangle$ states as: $\left|\alpha_{P}\right\rangle=\sum_{k} P|k\rangle a_{k}^{\alpha_{P}}$. We now act on this equation with $\left\langle\alpha_{Q}\right| Q \omega P$ where $\left\langle\alpha_{Q}\right|$ is an arbitrary state in the $Q$ space. Using eq. 1 we have:

$$
\begin{aligned}
\left\langle\alpha_{Q}|Q \omega P| \alpha_{P}\right\rangle & =\sum_{k}\left\langle\alpha_{Q}|Q \omega P| k\right\rangle a_{k}^{\alpha_{P}} \\
& =\sum_{k}\left\langle\alpha_{Q} \mid k\right\rangle a_{k}^{\alpha_{P}}
\end{aligned}
$$

Since the $\left|\alpha_{P}\right\rangle$ are an orthonormal set the expansion coefficients $a_{k}^{\alpha_{P}}$ can be determined by inverting the equation:

$$
\left\langle\alpha_{P}^{\prime} \mid \alpha_{P}\right\rangle=\delta_{\alpha_{P}^{\prime} \alpha_{P}}=\sum_{k}\left\langle\alpha_{P}^{\prime} \mid k\right\rangle a_{k}^{\alpha_{P}} .
$$

This is the defining equation of the Lee-Suzuki method. Once this equation is solved we have $\omega$ and can calculate the effective interactions and operators. However the inverse of $\left\langle\alpha_{P}^{\prime} \mid k\right\rangle$ must exist. If not you have to choose a different set of $|k\rangle$ 's or modify the projection operator $P$. The existence of the inverse implies that there are the same number of states in the $P$ space and the set $|k\rangle$.

The oddity of the Lee-Suzuki method is that $\omega$ depends not only on $P$ but also on the states chosen to be in the set $|k\rangle$. For example, even if you are only interested in the lowest state, the $\omega$ acting on it will change if the set $|k\rangle$ is modified to include the 101st state rather than the 100th.

Note the dramatic differences compared to the previous method. Much to the surprise of people use to working with the Bloch-Horowitz or Feshbach method the effective operators in this method are energy independent. Also this method can work for no more states than the number of states in the $P$ space. This is also in distinct contrast to the Bloch-Horowitz method.

Since the $V_{\text {low } k}$ obtained in the renormalization group method is equivalent to the Lee-Suzuki method you might expect that a matrix inversion like that defined in eq. 15] would also play an important role in the renormalization group derivation. Indeed this is the case, see for example eq. 4 and 5 in ref. [7]. The renormalization group $V_{\text {low } k}$ is given by eq. 9 with current choice of $\omega$.

\section{UNITARY TRANSFORMATIONS}

It is well known that the Lee-Suzuki method corresponds to a unitary transformation $[9]$ and eq. 7 does suggests a unitary transformation with:

$$
U=\frac{1}{\sqrt{1+P \omega^{\dagger} Q \omega P}}\left(P+P \omega^{\dagger} Q\right) .
$$

However, $U$ is not a unitary transformation since $U U^{\dagger}=P$ rather than one. What $U$ does is take a state in the $|k\rangle$ space and transform it into the $P$ space while $U^{\dagger}$ does the inverse, namely maps a state in the $P$ space into a state in the $|k\rangle$ space. The operator, $U$, acting on a state in the $\left|k^{\prime}\right\rangle$ space gives zero. A full unitary operator would have to map states in $\left|k^{\prime}\right\rangle$ into the $Q$ space. An operator that does this is:

$$
\begin{aligned}
\mathcal{U}= & \frac{1}{\sqrt{1+P \omega^{\dagger} Q \omega P}}\left(P+P \omega^{\dagger} Q\right) \\
& +\frac{1}{\sqrt{1+Q \omega P \omega^{\dagger} Q}}(Q-Q \omega P) \\
= & \frac{1-\left(Q \omega P-P \omega^{\dagger} Q\right)}{\sqrt{1+P \omega^{\dagger} Q \omega P+Q \omega P \omega^{\dagger} Q}} .
\end{aligned}
$$

Note $\left(Q \omega P-P \omega^{\dagger} Q\right)^{2}=-\left(P \omega^{\dagger} Q \omega P+Q \omega P \omega^{\dagger} Q\right)$. When acting on a state in the $|k\rangle$ space this reduces identically to $U$ since $Q-Q \omega P$ acting on state in $|k\rangle$ gives zero. Thus if one is only interested in states in the $|k\rangle$ space then the only 
difference between the two operators is aesthetics and ink usage. The full form does indicate that what the Lee-Suzuki method is doing, either explicitly or implicitly (depending on the formulation) is constructing a unitary transformation that block diagonalizes the the Hamiltonian.

For a state in the set $|k\rangle$, eq 16 can be simplified. Using eq. 10 and the definition of $\phi$ (eq. 6) gives $U|k\rangle=\sqrt{1-P \omega^{\dagger} Q \omega P} P|k\rangle=\left|\phi_{k}\right\rangle$. Using the completeness of the $\left|\phi_{k}\right\rangle$ in the $P$ space $\left|\phi_{k}\right\rangle$ can be expanded as $\left|\phi_{k}\right\rangle=\sum_{l} P|l\rangle\langle k|P| l\rangle^{-1 / 2}$. In this form the $\left|\phi_{k}\right\rangle$ are manifestly orthonormal. The wave functions $P|k\rangle$ span the $P$ space but are not orthonormal. The $\left|\phi_{k}\right\rangle$ have been orthogonalized in a way that treats all the states in the $|k\rangle$ space symmetrically.

In the Bloch-Horowitz method it also possible to construct a unitary transformation however it is much less useful since $\omega$ depends on the energy of the state of under consideration and the unitary transformation would be different for each state.

\section{CONCLUSIONS}

The important quantity in determining effective interactions or operators is $\omega$. A projection operator loses information. It is the job of $\omega$ to restore the lost information. The parallel derivation of the Bloch-Horowitz and Lee-Suzuki methods shows that the essential difference between them is how the lost information is recovered or equivalently how $\omega$ is determined. The crucial step in the Bloch-Horowitz method is a partial solution of the Schrodinger equation while in the Lee-Suzuki method the key step is a matrix inversion.
While the main equations (3) through 6) in the two methods are similar there are significant differences due to the different methods of restoring the lost information. The Bloch-Horowitz method deals with one state at a time and has energy dependent effective interactions and operators. It can describe any state with a non-zero overlap with the model space. The Lee-Suzuki method deals with a set of wave functions, a set with the same dimension as the $P$ space. It can describe only those states in that set. The effective interactions and operators are energy independent.

Another method for using a restricted space is the renormalization group. The crucial equation in comparing to the renormalization group is eq. 3. It shows you how to construct a model-space effective operator that gives the same model-space components of the wave function as the calculation in the full space. Either the Bloch-Horowitz or Lee-Suzuki methods can be used. The first reproduces the fully off-shell t-matrix and is thus equivalent to the procedure of Birse et al. The second only reproduces the half-off shell t-matrix and is equivalent to $V_{\text {low } K}$.

\section{ACKNOWLEDGMENTS}

The author was motived by a seminar by B. Barrett at TRIUMF and benefited greatly from discussions during and following a seminar at the Institute for Nuclear Theory at the University of Washington. Useful discussion where held with B. Barrett, W. Haxton, and A. Schwenk. The Natural Sciences and Engineering Research Council of Canada is thanked for financial support.
[1] C. Bloch and J. Horowitz, Nucl. Phys. 8, 91 (1958).

[2] H. Feshbach, Ann. Phys. (N.Y.) 5, 357(1958); 19, 287 (1962); B.Block and H. Feshbach, ibid. 2347 (1963)

[3] M.C. Birse, J.A. McGovern and K.G. Richardson, Phys. Lett. B464 (1999) 169.

[4] S.Y. Lee and K. Suzuki, Phys. Lett. B91 (1980) 173; K. Suzuki and S.Y. Lee, Prog. Theor. Phys. 64 (1980) 2091.

[5] P. Navratil, B. R. Barrett, Phys. Rev. C54 (1996) 298.
[6] S.K. Bogner, T.T.S. Kuo, A. Schwenk, D.R. Entem, and R. Machleidt Phys. Lett. B576 265 (2003).

[7] S.K. Bogner, A. Schwenk, T.T.S. Kuo, and G.E. Brown, nuc1-th/0111042

[8] P. Navratil, G.P. Kamuntavicius, and B.R. Barrett, Phys. Rev. C61 (2000) 044001. b

[9] K. Suzuki, Prog. Theor. Phys. 68, 1999 (1982); K. Suzuki and R. Okamoto, Prog. Theor. Phys. 92, 1045 (1994). 